\title{
Emergency Medical Services Utilization during an Outbreak of Severe Acute Respiratory Syndrome (SARS) and the Incidence of SARS-associated Coronavirus Infection among Emergency Medical Technicians
}

\author{
Patrick Chow-In Ko, MD, Wen-Jone Chen, MD, PhD, \\ Matthew Huei-Ming Ma, MD, PhD, Wen-Chu Chiang, MD, Chan-Ping Su, MD, \\ Chien-Hua Huang, EMT-P, Tsung-Chien Lu, MD, Fuh-Yuan Shih, MD, \\ Fang-Yue Lin, MD, PhD
}

\begin{abstract}
Objectives: This was a study to evaluate the utilization of emergency medical services (EMS) systems during the outbreak of severe acute respiratory syndrome (SARS), and to assess the incidence of infection among emergency medical technicians (EMTs). Methods: This was a prospective, observational study conducted in the EMS system of Taipei, Taiwan. Probable/suspect cases of SARS were defined by World Health Organization criteria. SARS-related transports were categorized into 1) requests from hospitals for probable/suspect cases of SARS, 2) quarantined individuals, and 3) febrile persons. City ambulances were organized into teams A, B, and C for transports of different perceived risks. Data on the EMS volume, the transport category, the final SARS status of patients, and the EMT responsible for the transports were collected. The EMS projected volume was computed by previous years' data and compared with that collected. The SARS incidence among EMTs was assessed by investigating probable SARS (P-SARS) and by surveying the seroprevalence of SARSassociated coronavirus (SARS-CoV) antibody. Results: From March 18 to June 19, 2003, there were 7,961 EMS transports, similar to the volume projected from previous years $(7,506)$ ( $95 \% \mathrm{CI}=6,688$ to 8,324$)$. Of these, $1,760(22.1 \%)$ were SARSrelated. When SARS-related transports were excluded, there was a $12.2 \%$ decrease $(95 \% \mathrm{CI}=11.4 \%$ to $12.9 \%)$ in EMS activities. Requests from hospitals, quarantined individuals, and febrile citizens accounted for $23 \%, 18 \%$, and $59 \%$ of
\end{abstract}

SARS-related transports. Among the 397 P-SARS cases in the city of 2.65 million people (incidence $0.01 \%$, 95\% $\mathrm{CI}=0.01 \%$ to $0.02 \%), 138(35 \%)$ required EMS transports. Two EMTs working in team C, the team with the lowest risk, developed P-SARS. One of them died soon thereafter. The incidence of P-SARS was $0.6 \%(95 \% \mathrm{CI}=0.2 \%$ to $2.2 \%)$, or $0.1 \%(95 \% \mathrm{CI}=0.03 \%$ to $0.4 \%)$ per transport. SARS-CoV serology was available in $74.1 \%$ of EMTs who were alive. In addition to the surviving P-SARS EMT, one EMT from team $\mathrm{A}$, the team with the highest risk, was seropositive. Combining P-SARS and the seropositive case, three EMTs were infected (incidence $1.3 \%, 95 \% \mathrm{CI}=0.4 \%$ to $3.6 \%$ ). No patient transported by the infected EMTs developed SARS. The hospitals serving EMS by the infected EMTs had been involved in a clustered outbreak prior to the EMTs' infections. Conclusions: During the outbreak of SARS, the overall EMS volume did not change significantly, but the non-SARS EMS activities decreased. Compared with the general population, EMS providers are at higher risk of contracting the SARS virus regardless of different perceived levels of risk. Standard protections and procedures for infection control should be strictly followed during transport and within the hospital environment. Key words: emergency medical services; severe acute respiratory syndrome; emergency medical technicians; utilization; incidence. ACADEMIC EMERGENCY MEDICINE 2004; 11:903-911.
From the Department of Emergency Medicine, National Taiwan University Hospital, College of Medicine, National Taiwan University (PC-IK, W-JC, MH-MM, W-CC, C-PS, T-CL, F-YS, F-YL), Taipei, Taiwan; and Medical Director Committee, Taipei Fire Department (C-HH), Taipei, Taiwan.

Received January 6, 2004; revision received March 8, 2004; accepted March 24, 2004.

Address for correspondence and reprints: Matthew Huei-Ming Ma, MD, PhD, No. 7, Chung-Shan S. Road, Department of Emergency Medicine, National Taiwan University Hospital, Taipei 100, Taiwan. Fax: 886-2-23223150; e-mail: mattma@ha.mc.ntu.edu.tw.

Another SARS article appears on page 973.

doi:10.1197/j.aem.2004.03.016
Severe acute respiratory syndrome (SARS), a respiratory illness caused by a novel coronavirus (SARS$\mathrm{CoV}),{ }^{1,2}$ was first recognized in February 2003 and became epidemic in Asia. ${ }^{3}$ The disease has high potential to be transmitted by droplets and close contacts. ${ }^{4}$ SARS is known to spread extensively among health care workers (HCWs) in various settings, ${ }^{5-7}$ and health care-associated transmission of SARS may further result in clustered outbreaks in the communities. ${ }^{8}$ Studies in Hong Kong and Toronto reported that $62 \%$ and $51 \%$ of their infected cases 
were HCWs, respectively, and the majority of them were physicians and nurses (59\% to $64 \%$ ). SARS infections among other HCWs were also reported, although these reports were much less detailed. ${ }^{5-9}$

During the SARS outbreak, emergency medical services (EMS) systems were used to move febrile patients to medical facilities for further assessments and care, ${ }^{10}$ and they were also responsible for the transport of the ill or quarantined people who had close contacts with SARS patients. It is possible that EMS personnel were exposed to the SARS virus. Up to this time, SARS infection among paramedics was only briefly reported without any systematic investigation. ${ }^{7}$ The use of EMS for SARS patients' transport as well as SARS infection among EMS providers should be evaluated. Information of this kind will be helpful in our prevention strategies for future outbreaks of SARS.

The objectives of this study were to evaluate the use of EMS systems during an outbreak of SARS and to assess the incidence of SARS infection among EMS providers and compare it with that of the general population in an Asian metropolitan area with an outbreak of SARS.

\section{METHODS}

Study Design. This was a prospective, observational study in an Asian metropolitan EMS system during the SARS outbreak. The institutional review boards of the city EMS committee approved the surveys in the study.

Study Setting and Population. The metropolitan EMS system under study has 2.65 million residents in an area of 272 square kilometers. The EMS configuration is a fire-based, single-tiered, basic life support (BLS) system. There are 41 ambulance squads staffed by 322 EMTs, each with at least 264 hours of training. Currently, only one ambulance squad in the city provides advanced life support (ALS) services.

Definitions of Probable/Suspect SARS Patients. Probable or suspect cases of SARS were diagnosed based on the case definitions recommended by World Health Organization (WHO) criteria. ${ }^{11,12}$ A case was excluded if an alternative diagnosis could fully explain the patient's illness.

Suspect SARS (S-SARS) includes the following: 1) patients with (after November 1, 2002) the symptoms of fever greater than $38^{\circ} \mathrm{C}$ and coughing and breathing difficulties, as well as one or more of the following exposures within the ten days prior to the onset of the symptoms: close contact with a person who is a suspect or probable case of SARS, history of recent travel to a SARS-affected area, and recent residence in a SARS-affected area; 2) patients who have died from unknown acute respiratory diseases after November
1,2002 , and have had the above-mentioned history of exposure within ten days prior to the development of symptoms.

Probable SARS (P-SARS): An S-SARS case with one of the following was defined as P-SARS: 1) radiographic evidence of pneumonia or respiratory distress syndrome (RDS) on chest radiograph, 2) one or more laboratory detection screens positive in SARS-CoVrelated tests, or 3) autopsy findings consistent with pathology of RDS without an identifiable cause.

From March to June 2003, a total of 664 P-SARS cases were reported in Taiwan. ${ }^{13}$ Among these PSARS cases, 397 patients $(60 \%)$ were from the largest metropolitan city, Taipei, especially after an outbreak in one of the municipal hospitals in late April. ${ }^{8}$ In response to the growing epidemic, measures were taken by the government to limit transmission of SARS, including widespread use of quarantine. ${ }^{14}$ Beginning March 18, 2003, people who had close contact with a SARS patient were quarantined for ten days. In addition, beginning April 28, travelers arriving on airplane flights from SARS-affected areas were also quarantined for ten days. ${ }^{14}$

Study Protocol. After the report of the first P-SARS case in Taipei, the city EMS organized a special system for the transport of SARS cases or patients of high risk. Details of the system are described below.

Categorization of Patient Transports. Conventionally, the city EMS system does not get involved in interhospital transports, which are carried out by hospital ambulances. However, during the SARS outbreak, EMS was required to be responsible for all SARSrelated patient transports, including interhospital transfers.

EMS dispatchers screened all EMS calls with a unified protocol to acquire information on the occurrence of fever, quarantine status, and any diagnosis of SARS. All patient transports were categorized into 1) requests from the hospitals for interhospital transports of P-SARS and S-SARS cases or other high-risk febrile patients, or for transporting recovered P-SARS and S-SARS patients home; 2) individuals under quarantine ${ }^{14}$; 3) febrile patients seeking emergency care (defined as those with subjective fever sensation, or objective fever $>38.0^{\circ} \mathrm{C}$ measured at home or by EMTs on first contact); and 4) other afebrile patients. Transports of the first three categories of patients were defined as SARS-related patient transports.

Organization of SARS-related Patient Transports. The use of EMS teams for SARS-related patient transports evolved with time and events during the SARS outbreak. In the early stage of the outbreak, the only ALS squad in the city (team A) was designated as the primary responding unit for all SARS-related patient transports. After the in-hospital outbreak of SARS on 
April 22 in the municipal hospital, ${ }^{8}$ citizens with fever seeking emergency care were asked to call EMS, and all EMS providers were required to measure the tympanic temperature of all patients on first contact. As the number of SARS-related transports escalated, seven additional BLS ambulance squads (team B) were recruited for transporting individuals under quarantine as well as febrile patients in case team A was not immediately available.

The other 33 ambulance squads in the city were designated as team $\mathrm{C}$, and responded to EMS calls from persons without fever or SARS contact histories. On occasions when neither team A nor team B was immediately available, the dispatch center would send a nearby ambulance squad from team $C$ to respond to the call. Whenever possible, teams $B$ and $C$ were excluded from the transport of P-SARS patients (Figure 1). All EMTs of the city EMS ambulances were required to monitor their health status by measuring their tympanic temperatures once a day, and to report any physical symptoms.

Measures for Personal Protection and Infection Control. During the outbreak, guidelines on protective equipment and procedures for infection control and precautions issued by the Centers for Disease Control and Prevention ${ }^{15}$ were adopted by the city EMS. All EMTs were required to undergo in-service training regarding the guidelines, and to follow them for all SARS-related patient transports. All ambulance squads had the same access to protective equipment and training in infection control procedures.

\section{Measurements.}

Overall EMS Utilization. The number of overall EMS transports during the study period was obtained and compared with the EMS volume from the same community in the previous year when there was no SARS incidence. The projected EMS volume in the same period was calculated using linear regression based on data for the previous four years.

SARS-related EMS Transports. The nurses in the dispatch center prospectively collected the EMS transports of each SARS-related patient category and traced the final diagnosis of these patients. The final diagnoses of P-SARS and S-SARS were made by the consensus of an expert SARS panel from the Taiwan Centers for Disease Control according to the case definitions (see above) recommended by the WHO. ${ }^{11,12}$ The consensus was made by reviewing all relevant clinical, epidemiologic, radiographic, and laboratory data. The date, the transport category, the ambulance team, and the EMTs responding to each transport were recorded. The date and the number of all P-SARS cases reported from the metropolitan area were also collected and compared with the daily amount of overall SARSrelated transports to evaluate their correlation in the same time frame.

\section{PATIENT CHARACTERISTICS DISPATCH PRIORITY OF RESPONSE TEAMS}
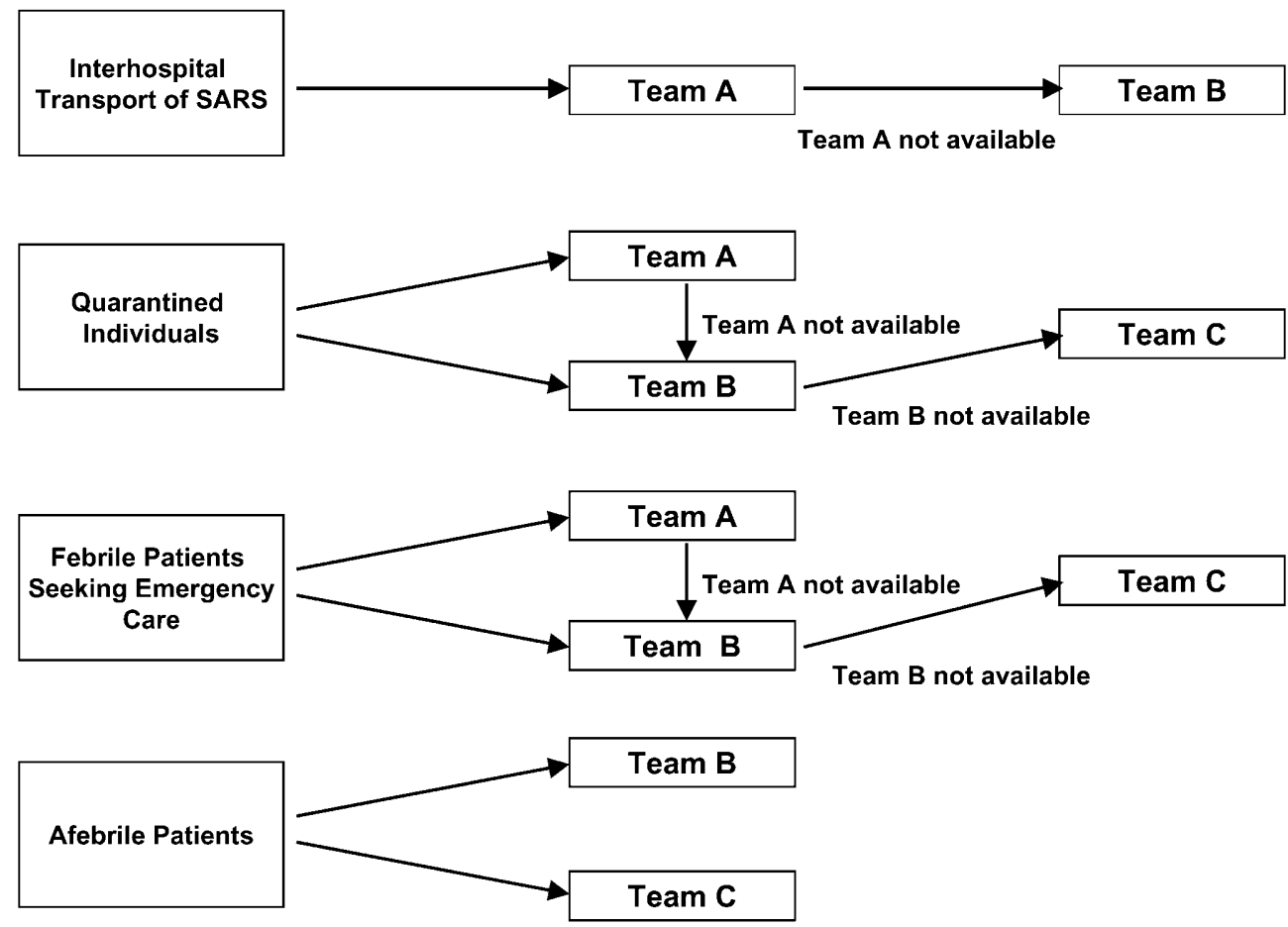

Figure 1. Schematic representation of EMS responses for severe acute respiratory syndrome (SARS)-related transports during the outbreak. 
Survey of SARS Infection among EMTs. In this study, SARS infections among EMTs were defined as 1) PSARS by WHO criteria confirmed by the consensus of the expert SARS panel from the Taiwan Center for Disease Control, ${ }^{11,12}$ or 2) a positive serology result of SARS-CoV antibody by indirect immunofluorescence assay (IFA) ${ }^{16}$ in asymptomatic individuals.

P-SARS of EMTs. EMTs with fever of more than $38^{\circ} \mathrm{C}$ were to report, receive a medical checkup, and be quarantined or admitted if necessary. The number of P-SARS and S-SARS cases among studied EMTs and diagnosed by the consensus of the expert panel was obtained from the Department of Health SARS patient registry in September 2003.

Seroprevalence of SARS-CoV Antibody. Blood samples of EMTs were obtained from July 15 to August 10, 2003, for seroprevalence survey. IFA on serum was used for detection of SARS-CoV antibody. ${ }^{16}$ As prior infection with SARS-CoV virus would have been exceedingly rare, a positive serology result is considered indicative of acute or recent infection with SARS$\mathrm{CoV}$ in a patient with a SARS-like illness. ${ }^{17}$ Previous research reported that nearly $100 \%$ of SARS patients had positive antibody response to SARS-CoV during the convalescent phase, and the SARS-specific immunoglobulin $G$ antibody persisted for more than 12 weeks. ${ }^{18}$ The reported sensitivity and specificity of IFA to the diagnosis of SARS were higher than $90 \%$ four weeks after the disease onset. ${ }^{19}$ These findings indicate that the profiles of antibodies to SARS might be helpful in epidemiologic surveys. In our study, serum samples were obtained four weeks after reporting the last probable case in the community, and 12 weeks after the initial outbreak. The time frame is considered to be appropriate for seroprevalence estimation.

The incidence of P-SARS and seroprevalence for SARS-CoV were reported and analyzed by the number of studied EMTs and by the number of transports made when appropriate. The incidence of P-SARS cases in the studied EMTs was also compared with that of the general population in the city.

Data Analysis. Data were entered, processed, and analyzed using SPSS for Windows (Release 10.0; SPSS Inc., Chicago, IL). Data were reported as the number of events or proportion unless otherwise specified; 95\% confidence interval ( $95 \% \mathrm{CI}$ ) of proportion was computed. Comparison was done using a t-test or Fisher's exact test where appropriate. All tests were two-tailed; $\mathrm{p}<0.05$ was accepted as statistically significant.

\section{RESULTS}

Utilization of EMS. From March 18 to June 18, 2003, there were a total of 7,961 city EMS transports. Among these, $1,760(22.1 \%)$ were SARS-related. None of the other 6,201 patients transported by EMS turned out to have SARS. Compared with the 7,059 EMS transports during the same period in 2002 , there was a $12.8 \%$ $(95 \% \mathrm{CI}=12.0 \%$ to $13.6 \%)$ increase in overall EMS volume, or a $12.2 \%(95 \% \mathrm{CI}=11.4 \%$ to $12.9 \%)$ decrease in EMS activities when excluding SARS-related transports. The projected EMS volume based on previous years was $7,506(95 \% \mathrm{CI}=6,688$ to 8,324$)$ transports. The overall number of actual EMS transports collected during the outbreak period was not overwhelming.

SARS-related EMS Transports. The date and the number of P-SARS cases reported from the metropolitan area are presented in Figure 2A. Most of the P-SARS cases were reported between late April and early June. Transports of patients from the hospitals, of quarantined individuals, and of febrile patients seeking emergency care accounted for $406(23 \%), 320$ $(18 \%)$, and $1,034(59 \%)$ of SARS-related transports, respectively. The date and the number of SARSrelated EMS transports are illustrated in Figure 2B. The requests for SARS-related EMS transports were closely associated with hospital-associated outbreaks and the epidemic curve of the disease. As seen in Figure 2, the daily transport volume started to escalate after the outbreak at the municipal hospital, reached the first peak about two weeks after the outbreak, and attained a second peak about four weeks after the initial outbreak. Team A responded to $54 \%$ of all SARS-related transports; team B and team C responded to $22 \%$ and $24 \%$ of SARS-related transports, respectively. The SARS-related EMS transports by transport categories and by transport teams are listed in Table 1. The numbers of cases that were finally diagnosed as P-SARS or S-SARS in each transport category are also presented in Table 1.

Among the 397 P-SARS cases reported in the metropolitan area, $138(35 \%, 95 \% \mathrm{CI}=30 \%$ to $40 \%)$ patients required EMS transport in the course of their treatment. When information on the final diagnoses of all transported patients was available, we were able to compare the perceived versus actual risks of SARS infection among all patient categories. Transports of patients from the hospitals posed a much higher threat of exposure to P-SARS or S-SARS when compared with the other two patient categories (Table 1). When transporting quarantined individuals versus febrile patients seeking emergency care, EMTs were confronted with similar risks of exposure to P-SARS $(4.4 \%$ vs. $2.6 \%$, $\mathrm{p}=0.13)$ or S-SARS $(2.8 \%$ vs. $3.2 \%, \mathrm{p}=0.85)$ patients.

\section{Incidence of SARS Infection among EMS Personnel.}

P-SARS and S-SARS of EMTs. Two EMTs were diagnosed as having P-SARS in early May. They had 
(A)

Probable SARS

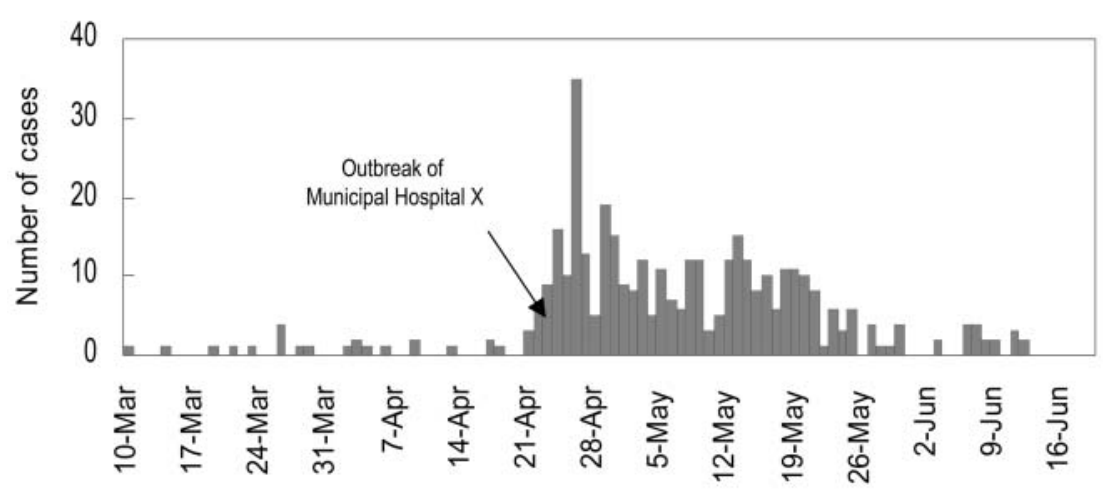

(B)

Febrile $\square$ Quarantined $\mathbf{~ I n t e r h o s p i t a l ~}$

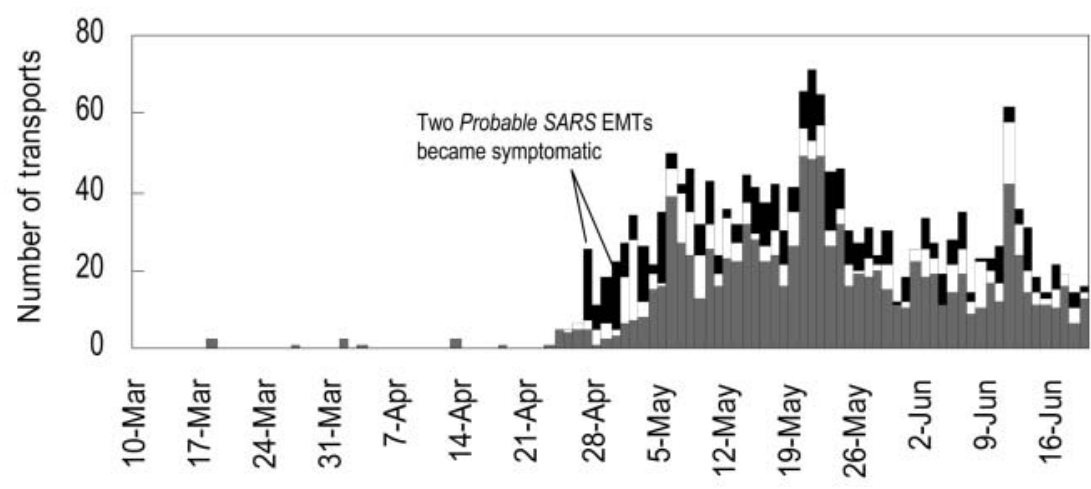

Figure 2. (A). The date and the number of probable severe acute respiratory syndrome (SARS) cases according to World Health Organization (WHO) criteria reported from the metropolitan city. (B). The date and the number of EMS transports for each patient category.

both worked in team C (Table 2). One EMT died soon thereafter. The other experienced acute respiratory distress syndrome and was supported with mechanical ventilation for almost one month. He survived and was discharged four months later. The incidence of P-SARS among all 322 EMTs was 0.6\% (2/322; 95\% $\mathrm{CI}=0.2 \%$ to $2.2 \%$ ) and the mortality rate was $0.3 \%$ $(1 / 322 ; 95 \% \mathrm{CI}=0.1 \%$ to $1.7 \%)$. The overall risk of P-SARS per SARS-related transport was $0.1 \%$ $(2 / 1,760 ; 95 \% \mathrm{CI}=0.03 \%$ to $0.4 \%)$. One EMT who worked in team B was diagnosed as having S-SARS. Although team A responded to the majority of transports of P-SARS cases from the hospitals, none of their staff developed P-SARS or S-SARS.

For the general population in the metropolitan area, the incidence of P-SARS was $0.01 \%$ (397 P-SARS among 2.65 million residents, $95 \% \mathrm{CI}=0.01 \%$ to $0.02 \%$ ).

Seroprevalence of SARS-CoV Antibody among EMS Personnel. Beside the EMT from team $C$ who died, SARS-CoV serology was investigated in $238(74.1 \%)$ of 321 EMTs, including all 87 EMTs from teams A and B, and $151(64.5 \%)$ of the 234 EMTs from team C (Table 2). Two EMTs tested positive $(2 / 238$, incidence: $0.8 \%$; $95 \% \mathrm{CI}=0.2 \%$ to $3.0 \%$ ) for SARS-CoV antibody, including the one from team $\mathrm{C}$ who survived P-SARS described above, and one EMT from team A. This additional seropositive EMT from team A did not develop fever greater than $38^{\circ} \mathrm{C}$ during the outbreak period; however, he did report tympanic temperature up to $37.7^{\circ} \mathrm{C}$ and rhinorrhea for two days in mid-May. He denied other associated symptoms of diarrhea, cough, or headache. The subclinical seropositive rate of SARS-CoV antibody among EMTs (i.e., excluding the ones already diagnosed as having P-SARS) was $0.4 \%(1 / 237 ; 95 \% \mathrm{CI}=0.1 \%$ to $2.4 \%)$.

Overall SARS Infection Rate. When we combined P-SARS cases and seropositive cases, a total of three EMTs were infected. The overall incidence of SARS infection was $1.3 \%(3 / 239 ; 95 \% \mathrm{CI}=0.4 \%$ to $3.6 \%)$ among the studied EMTs. Table 2 shows the number and the incidence of SARS infection among EMTs by EMS teams. 
TABLE 1. Severe Acute Respiratory Syndrome (SARS)-related EMS Transports by Transport Categories and by Transport Teams

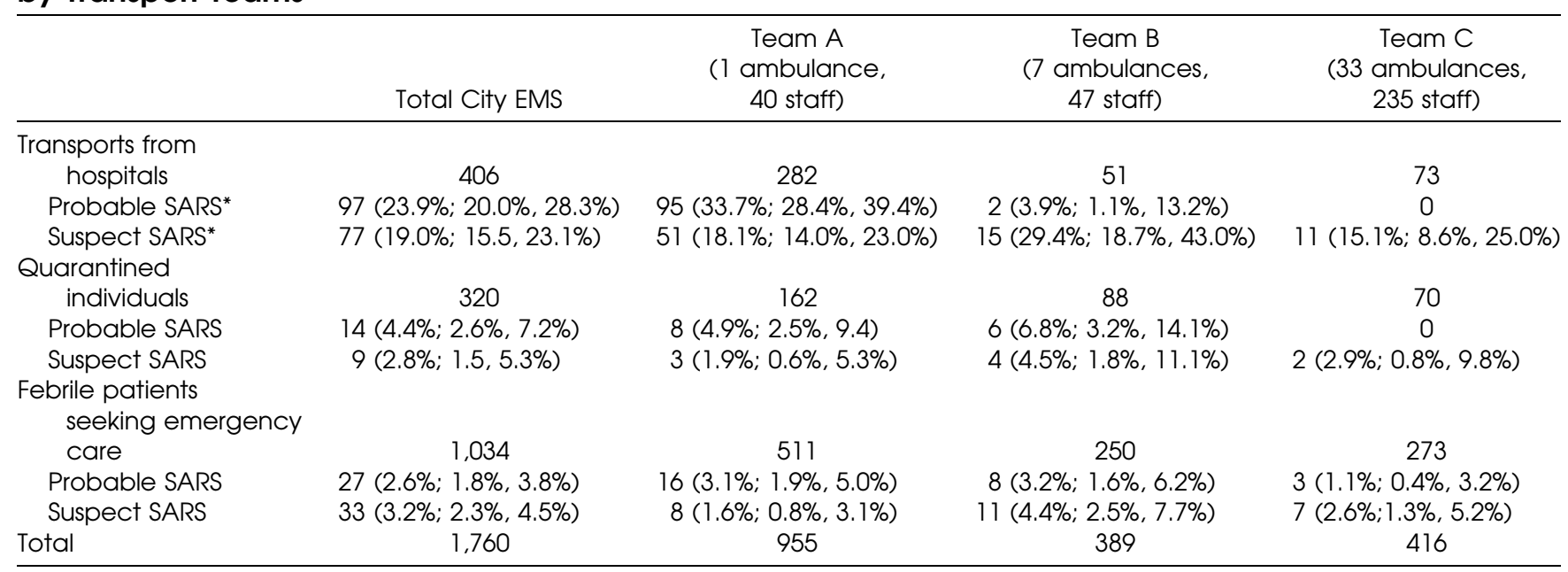

*Transports of recovered probable/suspect SARS were excluded.

Data are presented as numbers (proportions \%; 95\% confidence intervals).

After careful review, we noted that none of the patients transported by the three SARS-infected EMTs was diagnosed as having P-SARS or S-SARS. None of the families or close friends of the three infected EMTs had SARS. There was no interaction between the infected EMTs. The three hospitals routinely receiving EMS patients transported by the three SARS-infected EMTs were all involved in the secondary clustered transmission linked to the outbreak at the municipal hospital. ${ }^{8}$

\section{DISCUSSION}

Severe acute respiratory syndrome may strike with particular force at HCWs who provide first contact care to acutely ill patients, namely, providers of EMS and hospital emergency department staff. Evaluating SARS infection of an EMS system in a SARS endemic area would provide valuable information for future EMS response and preparedness for similar events. As a novel disease with tremendous threats to HCWs, SARS transmissions among EMS providers have not been thoroughly evaluated. This study is one of the first to evaluate EMS utilization during a SARS outbreak in a community, and to assess the incidence of P-SARS and seroprevalence among EMS providers.

Patients who have SARS may have a greater chance to spread the disease when they seek emergency care by taking public transportation such as the subway or taxis. In order to reduce the chance of disease transmission, professional EMS systems in many communities were utilized for SARS-related patient transports during the last outbreak. The data in our system showed that the increase of overall EMS transports during the SARS outbreak was similar to the expected annual increase. When excluding

TABLE 2. Number and Incidence of Probable Severe Acute Respiratory Syndrome (SARS) and Seropositive Cases among EMTs during an Outbreak

\begin{tabular}{|c|c|c|c|c|}
\hline & Team A & Team B & Team C & Total City EMS \\
\hline Number of ambulances & 1 & 7 & 33 & 41 \\
\hline Number of staff & 40 & 47 & 235 & 322 \\
\hline Transports of probable SARS & 119 & 16 & 3 & 138 \\
\hline Number of EMT with probable SARS & 0 & 0 & 2 & 2 \\
\hline Incidence* of EMT with probable SARS & 0 & 0 & $0.9(0.2,3.1)$ & $0.6(0.2,2.2)$ \\
\hline \multicolumn{5}{|c|}{ Risk $^{*}$ of probable SARS case per SARS-related } \\
\hline Number of seropositive EMT & 1 & 0 & 1 & 2 \\
\hline Incidence* of seropositive EMT & $2.5(0.4,12.9)$ & 0 & $0.7(0.1,3.7)$ & $0.8(0.2,3.0)$ \\
\hline Total SARS infectiont among EMT & 1 & 0 & 2 & 3 \\
\hline Incidence* of SARS infectiont of EMT & $2.5(0.4,12.9)$ & 0 & $1.3(0.4,4.7)$ & $1.3(0.4,3.6)$ \\
\hline
\end{tabular}

${ }^{*}$ Incidence/risk and (95\% confidence interval).

tSARS infection included probable SARS and seropositive cases. 
SARS-related transports, the amount of transports of other emergencies during the outbreak period was lower than that of the previous years. The general public may be reluctant to call EMS for fear of the potential of SARS transmission. Although the addition of SARS-related transports in the EMS system during the outbreak would not significantly affect the overall number of EMS transports, the logistic and psychological effects could be substantial and need to be addressed.

During the studied period, SARS-related EMS utilization totaled 1,760 transports representing $22.1 \%$ of all EMS activities, and was closely associated with ongoing clustered outbreaks in health care facilities and communities. The relation between the amount of SARS-related EMS utilization and the number of infected patients in the community should serve as a guideline for EMS need assessment in future outbreaks.

The majority of SARS-related transports were for febrile patients seeking emergency care, followed by transports requested from hospitals, and quarantined individuals. Transfer of patients from hospitals was not a usual routine in EMS services in the studied metropolitan area. As most community hospitals were stretched to their limit in taking care of febrile patients with maximal vigilance, the city authority recruited EMS to carry out transfers of P-SARS and S-SARS patients between hospitals. EMS was required to respond to the interhospital transfer when the hospitals were overcrowded or were inadequate to take care of SARS patients, or when asked to send the recovered SARS patients home. The ambulances of these community hospitals were used for interhospital transfer of only patients without fever or suspicion of SARS infection.

According to our data, about one third (138 of 397) of the probable SARS cases in the metropolitan area activated EMS transport in the course of their treatment; about $70 \%$ (97 of 138) of these were interhospital transfers, $20 \%$ (27 of 138) were febrile patients seeking emergency care, and $10 \%$ (14 of 138) were quarantined individuals. The incidences of P-SARS among quarantined individuals and febrile patients seeking emergency care were very similar $(4.4 \%$ vs. $2.6 \%$, Table 1). All EMTs should maintain the same standards of infection control and precautions when transporting both categories of patients.

The incidence rate of SARS infection among the EMTs we reported is $1.3 \%$. The rate is not as high as that of hepatitis B virus transmission among HCWs, which is reported to be between $2 \%$ and $40 \%,{ }^{20}$ but it is a little higher than that of the annual tuberculin conversion rate $(0.5 \%)$ of EMS personnel. ${ }^{21}$ However, considering the high case fatality ratio of P-SARS cases $(7 \%$ to $40 \%)$, and the significant consequence of secondary clustered transmission of $\mathrm{HCWs}$, ${ }^{8,22}$ EMS systems should spare no effort to reduce the occupa- tional transmission of SARS virus to the minimum. In our system, an attempt to reduce the risk of exposure among EMTs was made by designating special transport teams and categorizing SARS-related transports according to perceived levels of risks. The purpose of the original transport scheme was to try to contain the risk of exposure to SARS virus to a limited number of ambulance crews. However, because of the increased volume secondary to clustered outbreaks, and the city policy of transporting all febrile patients seeking emergency care by EMS, all the teams were ultimately exposed to P-SARS patients. Contrary to original expectations, the severely infected EMTs (i.e., P-SARS cases with respiratory distress) were all from team C, which was responsible for transporting patients with the lowest perceived risk of SARS. However, all three teams had the same access to protective equipment and received the same education and training in infection control procedures. It might be postulated that EMTs of this team (team C) had a false sense of security and did not follow the standard operation guidelines for personal protection during their transports, or had less incentive for education and training.

Detection of SARS-CoV antibody in a serum sample is one of the laboratory criteria for SARS. ${ }^{23}$ As prior infection with SARS-CoV virus would have been exceedingly rare before the outbreak in March 2003, a positive serology result could be indicative of acute or recent infection with SARS-CoV in a patient with a SARS-like illness. ${ }^{17}$ Beside EMTs with P-SARS, one relatively healthy EMT among the 237 tested positive for SARS-CoV antibody $(0.4 \% ; 95 \% \mathrm{CI}=0.1 \%$ to $2.4 \%)$. It implied the possible existence of subclinical or asymptomatic cases among HCWs. ${ }^{24-26}$ The incidence of P-SARS among EMTs studied in our metropolitan EMS system was $0.6 \%(2 / 322 ; 95 \% \mathrm{CI}=0.2 \%$ to $2.2 \%)$, well above that of the general public in this metropolitan area (incidence: $0.01 \%$; $95 \% \mathrm{CI}=0.01 \%$ to $0.02 \%$ ). Most individuals with SARS were HCWs, i.e., physicians and nurses. ${ }^{5-7,27}$ The incidence of SARS infection among physicians or nurses was not studied in this report. According to the data from other locales, among the $770 \mathrm{HCWs}$ who had contact with SARS patients, 18 (2.3\%) were infected with the virus. The respective rates of infection reported among nursing assistants, nurses, and physicians were $6.67 \%, 4.78 \%$, and $2.88 \%{ }^{28}$ It is reasonable to assume that EMS personnel who had contact with the SARS patients only briefly during the transport period were at lower risk of contracting SARS when compared with HCWs who had more frequent contacts with SARS patients. ${ }^{28}$

The sources of virus infections for the EMTs deserve closer examination. When all patients transported by the three SARS-infected EMTs were reviewed and followed-up, none was diagnosed as having P-SARS or S-SARS. Therefore, SARS transmission from direct patient contact during transport would be unlikely to 
explain the infection. None of the families or close friends of the three infected paramedics had SARS. Possible sources of infection for these EMTs included virus transmission from nonoccupational exposure to SARS cases in the community, from contacting unrecognized asymptomatic SARS cases during transport, and from touching contaminated surfaces in the hospital environment. Subclinical SARS cases in the community, if they existed, might not have had fever in their courses and might have remained unidentified during the outbreak. These patients might have infected the EMTs. Moreover, all three hospitals serving EMS patients transported by the three infected EMTs had been involved in the clustered transmission linked to the outbreak at the municipal hospital prior to the EMTs' onsets of illness. The infected EMTs might have been exposed to the virus in the hospital.

\section{LIMITATIONS}

There were several limitations to our study. First, it is limited by the observational design. Second, the triage scheme for SARS-related patient transports did not include febrile patients seeking emergency care until April 22, 2003, when the clustered outbreak in the municipal hospital occurred. That might have affected the risk estimation for different teams in our study. Third, we surveyed the seroprevalence of SARS-CoV antibody during the convalescent phase to investigate the possibility of subclinical infection, which should be included in the estimation of the incidence of EMT occupational infection. Acute sera of subclinical cases were not available because these patients were probably asymptomatic and would not be seeking medical care. Without the baseline sera before the outbreak or acute sera, we could not exclude the possibility of preceding infection of the seropositive cases before the outbreak, although it might be rare at present. Fourth, not all EMTs received the serology survey. Those not taking the serology test were all from team C. It was probable that they perceived themselves to have the lowest risk of infection and considered the survey unnecessary. This may cause the incidence of infection to be over- or underestimated.

\section{CONCLUSIONS}

While integrating SARS-related transports in EMS, the overall transports would not significantly increase, and the general public would be reluctant to use EMS during a SARS outbreak. Utilization of EMS for transporting SARS-related patients in an outbreak was closely associated with hospital-associated outbreaks and the epidemic curve of the disease. Compared with the general population, EMS providers are at a higher risk of contracting SARS virus regardless of different perceived levels of risk. Standard protections and procedures for infection control should be strictly followed during both SARS-related transports and inhospital periods.

The authors appreciate the contributions of all EMTs of the city EMS who showed their bravery and devoted themselves in the fight against SARS. Thanks also to the staff of the ALS ambulance team who helped with the implementation of the seroprevalence survey.

\section{References}

1. Peiris JS, Lai ST, Poon LL, et al. Coronavirus as a possible cause of severe acute respiratory syndrome. Lancet. 2003; 361:1319-25.

2. Drosten C, Gunther S, Preiser W, et al. Identification of a novel coronavirus in patients with severe acute respiratory syndrome. N Engl J Med. 2003; 348:1967-76.

3. Benitez MA. Hong Kong bears brunt of latest outbreak [news]. Lancet. 2003; 361:1018.

4. Centers for Disease Control and Prevention (CDC). Basic information about SARS, September 29, 2003. Available at: http: //www.cdc.gov/ncidod/sars/factsheet.htm. Accessed Oct 10, 2003.

5. SARS Investigative Team, CDC. Cluster of severe acute respiratory syndrome cases among protected health-care workers-Toronto, Canada, April 2003. JAMA. 2003; 289:2788-9.

6. Lee $\mathrm{N}$, Hui $\mathrm{D}, \mathrm{Wu}$ A, et al. A major outbreak of severe acute respiratory syndrome in Hong Kong. N Engl J Med. 2003; 348: 1986-94.

7. Booth CM, Matukas LM, Tomlinson GA, et al. Clinical features and short-term outcomes of 144 patients with SARS in the Greater Toronto Area. JAMA. 2003; 289:2801-9.

8. Centers for Disease Control and Prevention (CDC). Severe acute respiratory syndrome-Taiwan, 2003. MMWR Morb Mortal Wkly Rep. 2003; 52:461-6.

9. Koh D, Lim MK, Chia SE. SARS: health care work can be hazardous to health. Occup Med. 2003; 53:241-3.

10. Centers for Disease Control and Prevention (CDC). Updated interim guidance: pre-hospital emergency medical care and ground transport of suspected severe acute respiratory syndrome patients. April 29, 2003. Available at: http:/ / www.cdc.gov/ncidod/sars/emtguidance.htm. Accessed Dec 10, 2003.

11. World Health Organization (WHO), Geneva. 2003. Case definitions for surveillance of severe acute respiratory syndrome (SARS). Available at: http://www.who.int/csr/ sars/casedefinition/en/. Accessed Oct 11, 2003.

12. Center for Disease Control, Department of Health, Taiwan, ROC. Medical professionals: SARS case definition-definition of Taiwan SARS cases, May 15, 2003. Available at: http://www.cdc.gov.tw/sarsen/. Accessed Feb 14, 2004.

13. Center for Disease Control. Department of Health, Taiwan, ROC. Latest information: SARS data analysis-Taiwan. Available at: http://www.cdc.gov.tw/sarsen/. Accessed Dec 10, 2003.

14. Centers for Disease Control and Prevention (CDC). Use of quarantine to prevent transmission of severe acute respiratory syndrome-Taiwan, 2003. MMWR Morb Mortal Wkly Rep. 2003; 52:680-3.

15. Bolyard EA, Tablan OC, Williams WW, Pearson ML, Shapiro $\mathrm{CN}$, Deitchmann SD. Guideline for infection control in healthcare personnel, 1998. Hospital Infection Control Practices Advisory Committee. Infect Control Hosp Epidemiol. 1998; 19: 407-63.

16. World Health Organization (WHO), Geneva. 2003. Use of laboratory methods for SARS diagnosis. Available at: 
http://www.who.int/csr/sars/labmethods/en/\#lab.

Accessed Dec 29, 2003.

17. Centers for Disease Control and Prevention (CDC). Fact sheet: SARS laboratory diagnostics. September 26, 2003. Available at: http://www.cdc.gov/ncidod/sars/labdiagnosis.htm. Accessed Oct 15, 2003.

18. Li G, Chen X, Xu A. Profile of specific antibodies to the SARS-associated coronavirus. N Engl J Med. 2003; 349:508-9.

19. Fang LQ, Zhang PH, Yang BA, et al. The application of indirect immuno-fluorescence assay in the diagnosis of severe acute respiratory syndrome. Zhonghua Liu Xing Bing Xue Za Zhi. 2003; 24:484-6.

20. Gerberding JL. Management of occupational exposures to blood-borne viruses. N Engl J Med. 1995; 332:444-51.

21. Prezant DJ, Kelly KJ, Mineo FP, et al. Tuberculin skin test conversion rates in New York City emergency medical service health care workers. Ann Emerg Med. 1998; 32:208-13.

22. World Health Organization (WHO), Geneva. 2003. Summary of probable SARS cases with onset of illness from 1 November 2002 to 31 July 2003. September 26, 2003. Available at: http://www.who.int/csr/sars/country/table2003_09_23/en/. Accessed Dec 29, 2003.

23. Centers for Disease Control and Prevention (CDC). Updated interim U.S. case definition for severe acute respiratory syndrome (SARS). December 12, 2003. Available at: http://www.cdc.gov/ncidod/sars/casedefinition.htm. Accessed Dec 29, 2003.

24. Parry J. Asymptomatic animal traders prove positive for SARS virus [news]. BMJ. 2003; 327:582.

25. Chan PK, Ip M, Ng KC, et al. Severe acute respiratory syndrome-associated coronavirus infection. Emerg Infect Dis. 2003; 9:1453-4.

26. Xu G, Lu H, Li J, et al. Primary investigation on the changing mode of plasma specific IgG antibody in SARS patients and their physicians and nurses. Beijing Da Xue Xue Bao. 2003; 35(suppl):23-5.

27. Wu W, Wang J, Liu P, et al. A hospital outbreak of severe acute respiratory syndrome in Guangzhou, China. Chin Med J. 2003; 116:811-8.

28. Li L, Cheng S, Gu J. SARS infection among health care workers in Beijing, China. JAMA. 2003; 290:2662-3. 